\title{
Verification Method of Key-Exchange Protocols With a Small Amount of Input Using TAMARin Prover
}

\author{
Misato Nakabayashi \\ NTT Secure Platform Laboratories \\ Tokyo, Japan \\ misato.nakabayashi.mu@hco.ntt.co.jp
}

\author{
Yuki Okano \\ NTT Secure Platform Laboratories \\ Tokyo, Japan \\ yuki.okano.te@hco.ntt.co.jp
}

\begin{abstract}
We propose an automatic verification method for cryptographic protocols. Our method can verify whether or not the key-exchange protocol satisfies main security properties, requiring only the protocol specification as its input. The specification consists of, for example, how session keys are computed and which party sends/receives which data to/from the communication channel. We materialize our method using the TAMARIN prover, which is an automatic verification tool for cryptographic protocols. To show the validity of our method, we verify main security properties of several existing protocols and find that the results coincide with what are theoretically known in cryptography. Our method is effective, because the complexity of cryptographic protocols has significantly increased in recent years. To overcome the complexity, people have started using automatic verification tools to verify the security of cryptographic protocols, but existing tools have two problems. First, the user must be familiar with the details of cryptography in order to describe formally the security model as part of input. Second, the input code is complex and its creation is error-prone; protocol specification and security model must be written in a specific language together with protocol-dependent extra codes. These two problems are resolved by our method.
\end{abstract}

\section{CCS CONCEPTS}

- Security and privacy $\rightarrow$ Formal security models; Logic and verification; • Theory of computation $\rightarrow$ Verification by model checking.

\section{KEYWORDS}

formal verification; cryptographic protocols; Tamarin prover; keyexchange protocols; automatic verification

\section{ACM Reference Format:}

Misato Nakabayashi and Yuki Okano. 2021. Verification Method of KeyExchange Protocols With a Small Amount of Input Using TAMARIN Prover. In Proceedings of the 2021 International Symposium on Advanced Security on Software and Systems (ASSS '21), June 7, 2021, Virtual Event, Hong Kong. ACM, New York, NY, USA, 8 pages. https://doi.org/10.1145/3457340. 3458301

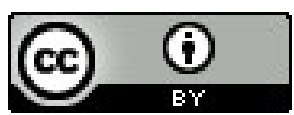

This work is licensed under a Creative Commons Attribution International 4.0 License ASSS '21, June 7, 2021, Virtual Event, Hong Kong. (C) 2021 Copyright held by the owner/author(s). ACM ISBN 978-1-4503-8403-2/21/06.

https://doi.org/10.1145/3457340.3458301

\section{INTRODUCTION}

The responses of governments and businesses to the COVID-19 Pandemic have led to changes in communication and working styles worldwide. People are forced to have meetings remotely, and confidential information previously communicated in face-to-face meetings is now communicated through systems. Therefore, the security of these systems has become more important.

Unfortunately, existing systems have experienced problems related to the vulnerability of cryptographic protocols. For example, in 2020, Marczak et al. [28] pointed out the inadequacy of end-toend encryption system in the videotelephony software program Zoom $[19,20]$. The problem is that while Zoom claims to provide end-to-end encrypted communication, it may actually be sending the secret key needed to decrypt the communication content to the server managed by Zoom.

System vulnerabilities are caused, for example, by design errors in cryptographic protocols. In the Zoom example above, a design error in the cryptographic protocol allowed a vulnerability to enter the system. The reason for this is that the security of cryptographic protocols is difficult to verify. The security verification of cryptographic protocols is not only complex, difficult, and error-prone but also requires a deep knowledge of cryptographic theory.

Therefore, for cryptographic protocols, a verification method is needed that prevents protocol designers from introducing errors and does not require the designers to know much about cryptographic theory. Such a verification method will be able to avoid system vulnerabilities caused by vulnerability of cryptographic protocols. Cryptographic protocols should preferably be verified at an earlier stage in the system development, i.e., the design phase, for two reasons. First, if a vulnerability is contained due to a mistake in the design phase, the security of the system cannot be guaranteed even if the implementation and operation are done correctly afterwards. Second, the later a mistake is detected, the more rework is required.

In summary, for cryptographic protocols, a verification method is needed that enables protocol designers to verify protocols in the design phase, prevents the designers from introducing errors, and can be used by designers with little knowledge of cryptographic theory. These three conditions should be satisfied by a technique called formal verification. Formal verification for cryptographic protocols describes the protocol and the security model (adversary model and security requirements) in a formal language and mathematically analyzes whether the protocol satisfies the security model or not. Indeed, in formal verification, the security of protocols is verified on the basis of the protocol specification, so implementation is not necessary and verification can be performed 
in the design phase. The inference during verification is done automatically by the computer, which avoids the introduction of errors. In addition, protocol designers can verify the security of protocols by only inputting the protocol and the security model, so they do not need to be familiar with cryptographic theory.

There already exist automatic formal verification tools that automatically obtain verification results, such as ProVerif [7-9], Scyther [12, 16], TAMARIN prover [3, 29, 31] and Verifpal [23, 25]. For details and comparison of automatic formal verification tools, see the survey by Barbosa et al. [2]. In the past, these tools successfully found security defects in various protocols, the most famous example is TLS 1.3 verification $[6,14,15]$.The Signal protocol [1,24], 5G authentication protocols [4, 13, 37, 38], and the QUIC protocol $[39,40]$ have also been verified using these tools.

However, the existing formal verification tools only partially satisfy the above three conditions. Existing tools satisfy the condition of enabling protocol designers to verify the security of a protocol in the design phase. In contrast, for the condition of preventing the designers from introducing errors, the input is complex and its creation is error-prone. To use existing tools, the protocol specification and security model must be written in a specific language (we call these descriptions protocol code and security model code, respectively). Originally, the protocol code should preferably consist only of the protocol specification, but extra information not included in the protocol specification needs to be described. As a result, the input becomes more complex, and errors are more likely to occur when creating the input. In addition, because the protocol code and the security model code are not independent of each other, a security model code needs to be created for each protocol. This requires some knowledge of cryptographic theory, so the condition of not requiring the designers to know about cryptographic theory is not completely satisfied. The input-process-output diagram of existing formal verification tools is shown in Figure 1.

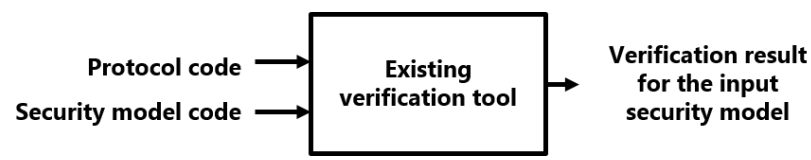

Figure 1: Input-process-output diagram of existing formal verification tools.

\subsection{Our Contributions}

In this paper, we propose an automatic verification method that fully satisfies the above three conditions. Our verification method uses the TAMARIN prover and can verify whether or not the protocol satisfies the main security properties, requiring only the protocol specification as its input. The specification consists of, for example, how session keys are computed and which party sends/receives which data to/from the communication channel. Moreover, to validate our verification method, we verify security properties of several existing protocols and show that the results coincide with results that are theoretically known in cryptography. Our verification method targets key-exchange protocols, which are a type of cryptographic protocols.
By using our verification method, designers can verify the security of protocols with less input. In particular, our method enables designers to verify the security of a cryptographic protocol with only the protocol specification, so that even people who are not familiar with cryptographic theory can verify the security of a protocol. Therefore, our method is expected to reduce the amount of errors, necessary knowledge, and effort in using formal verification tools.

The input-process-output diagram of our verification method is shown in Figure 2. Note that our method targets key-exchange protocols.

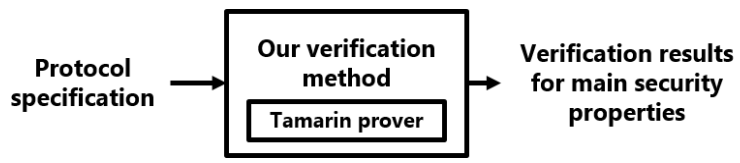

Figure 2: Input-process-output diagram of our proposed verification method.

\subsection{Related Work}

In this section, we review several existing studies on the ease of use of formal verification tools. Existing research has focused mainly on the input of protocol specification. There are still problems for the input of security properties.

Verifpal [23, 25] is an automatic verification tool for cryptographic protocols developed by Kobeissi et al. that is both innovative and amazing. Its input is very intuitive, and Verifpal is also designed to reduce user input errors. In particular, it is able to verify confidentiality and authentication security properties using natural language expressions such as "confidentiality?" and "authentication? Alice -> Bob". If a protocol designer wants to verify complex security properties (e.g., confidentiality after secret information is leaked), he/she needs to describe the information required for verification other than the protocol specification.

Keller [21] defined a cryptographic protocol description language that is independent of the verification tool and developed a program that automatically translated that language into the TAMARIN prover input code. This protocol description language is similar to the so-called Alice and Bob notation, which describes the messages exchanged between protocol parties during protocol execution, thus reducing the effort of creating the input for TAMARIN. In his research, Keller focused on simplifying the protocol input, and the only security models that can be easily verified (by simply copying and pasting existing security model code) are simple properties such as confidentiality of the secret information.

Shen et al. [32] defined a modeling method for cryptographic protocols based on a unified modeling language (UML) framework and proposed an easy-to-understand way of representing protocols using sequence diagram. Then, they devised a method to automatically convert the sequence diagram into input for ProVerif. In their research, they focused only on simple security properties such as the confidentiality of the secret information and the authentication of the communicating parties. 


\section{PRELIMINARIES}

In this section, we describe the key-exchange protocol that is the target of our verification method and the TAMARIN prover used in our verification method.

\subsection{Key-Exchange Protocols}

A key-exchange (KE) protocol is a protocol for sharing a session key, which is a common secret key, with a communicating party over an unreliable communication channel such as the Internet [10].

2.1.1 Several Concepts of KE Protocols. The executor of a protocol is called a party, and one action of executing the party's protocol (from the start of message exchange to the establishment of the session key) is called a session. Here, there are two parties: the initiator, who starts the session, and the responder, who is the communication partner of the initiator. Each session is assigned a unique identifier called a session ID, and each party is assigned a party ID, a long-term secret key, and a public key corresponding to the long-term secret key. Additionally, ephemeral secret information (e.g., random numbers generated in the session) used by each party in the protocol is called the ephemeral secret key.

As an example of a KE protocol, the Diffie-Hellman (DH) KE protocol [17] is shown in Figure 3. This is a KE protocol between two parties, Alice (initiator) and Bob (responder), which establishes a session key $g^{x y}$ using their respective ephemeral secret keys $x, y$.

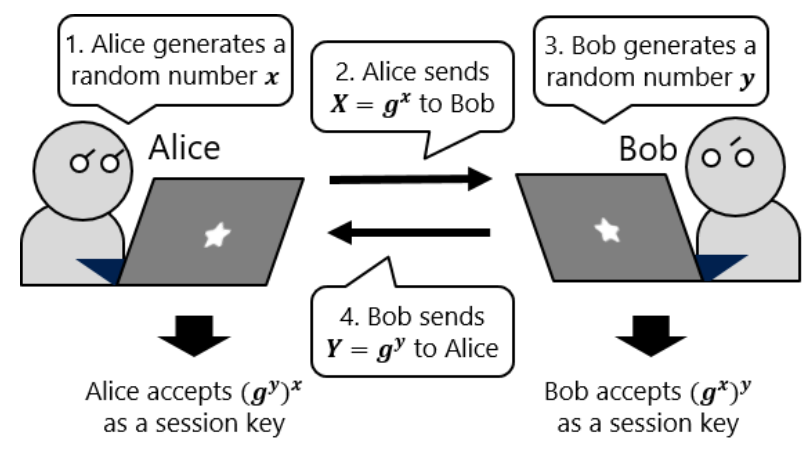

Figure 3: The Diffie-Hellman key-exchange protocol.

2.1.2 Security Model of KE Protocols. The security model of a KE protocol is defined by the abilities of an adversary and the conditions for security. There are two types of adversaries: passive adversaries who can only eavesdrop on messages flowing in the communication channel, and active adversaries who can eavesdrop, tamper, delete, and resend messages flowing in the communication channel. In addition to these abilities, it is assumed that the adversaries can obtain public information such as parity IDs and public keys, as well as session key information used in different sessions of the protocol. Furthermore, the long-term and ephemeral secret keys of a party and its communication partner are often assumed to be leaked. In this case, the situation where the session key can obviously be calculated (e.g., the adversary also has all the information held by the party) is excluded. For each session, a notion called a matching session is introduced to identify the party with which it is communicating. A matching session is a session that communicates with a session executed by a party and has equal input and output.

The main security properties are the confidentiality of the session key, forward secrecy and weak forward secrecy. Forward secrecy is a security property for an active adversary, which states that even if a party's long-term secret keys are leaked to the adversary at some point, the session key derived before the leak guarantees confidential. Moreover, weak forward secrecy is forward secrecy against a passive adversary.

\subsection{The Tamarin Prover}

2.2.1 Overview of TAMARIN Prover. The TAMARIN prover is a stateof-the-art cryptographic protocol verification tool developed by Meier et al. [3, 29, 31]. In the past, TAMARIN successfully found security defects in various protocols $[4,14,15,22,35,36]$. TAMARIN can verify security verification for the Dolev-Yao model [18], which symbolically models the execution of a protocol and the behavior of an adversary. In the Dolev-Yao model, key information and protocol messages flowing in the communication channel are treated as terms rather than strings, and cryptographic primitives such as a public key cryptography system are formalized as ideal, i.e., ciphertext can be decrypted only if who has the decryption key (i.e., without the decryption key, no plaintext information can be obtained). On top of that, the adversary is modeled as an active adversary who can eavesdrop, tamper, delete, and resend any message flowing in the communication channel.

In TAMARIN, a language based on multiset rewriting rules [11] is used to model the behavior of parties in a protocol and the information that an adversary can obtain. Then, execution traces are created on the basis of the described rules, and a security judgment is made for all the execution traces. Depending on the judgment result, one of the following is output: verified (no attack found), falsified (attack found), or incomplete (verification incomplete).

Compared with other automatic verification tools, TAMARIN has the advantage that the security model can be described intuitively. The security model of TAMARIN can easily describe complex security properties using logical formulas. Furthermore, TAMARIN can verify multiple security models at the same time.

2.2.2 Input for TAMARIN Prover. TAMARIN requires two types of input: protocol code, which models the behavior performed by the parties in the protocol (e.g., initiator or server), and security model code, which models the information that an adversary can obtain and the security property. The protocol code is written in a language based on multiset rewriting rules, and the security model code is written by using the same language as the protocol code and first-order predicate logic formulas. For detailed information on how to write TAMARIN input code, please refer to the official manual [33]. As an example of input of TAMARIN, the protocol code of the DH key-exchange protocol (see Figure 3) and the corresponding security model code for confidentiality of the session key when a party's ephemeral secret key is leaked by an adversary are shown in Appendix A.

2.2.3 Multiset Rewriting Rules and Facts. In TAmarin, the behavior of the protocol and the leakage of information to the adversary 
are described using multiset rewriting rules. A multiset rewriting rule is written in the following form.

$$
\begin{array}{ll}
1 & \text { rule Name: } \\
2 & {[\operatorname{Pre}(x)]--[\operatorname{Action}(x)]->[\operatorname{Post}(x)]}
\end{array}
$$

Each rule is applied when the current system state contains $\operatorname{Pre}(x)$, and when it is applied, $\operatorname{Pre}(x)$ is removed from the system state and Post $(x)$ is added. Then, Action $(x)$ is added to the execution trace. Here, Pre $(x)$, Action $(x)$, Post $(x)$ in the rule is called a fact, and the fact in the left parenthesis of the rule is called the Pre condition, the fact in the middle parenthesis is called the Action condition, and the fact in the right parenthesis is called the Post condition. The facts written in the Action condition are used to label the behavior and are called action facts. Action facts are used to describe the security model code.

\section{OUR PROPOSED METHOD}

In this section, we propose a verification method. Our verification method satisfies the following conditions: the only input is the protocol specification, and it can verify the main security properties of KE protocols. KE protocols are the most fundamental of all cryptographic protocols and are of great social importance. For this reason, we focus on the verification method of KE protocols. To realize this verification method, we introduce an algorithm to construct the protocol code of TAMARIN from protocol specification. This algorithm automatically adds the extra information needed for verification in TAMARIN. In addition, we show how we write a reusable (i.e., applicable to any protocol) security model code and create reusable security model codes for the main security properties of the KE protocol. Figure 4 shows a conceptual diagram of our verification method.

\subsection{Main Security Properties of KE Protocols}

The security model of KE protocols is defined by the abilities of the adversary and the security conditions, as described in Section 2.1.2. In our verification method, we assume that the adversary is an active one, and the verification properties are session key confidentiality when various information is leaked to the adversary, forward secrecy, and weak forward secrecy. For the confidentiality of the session key, we assume that the party's long-term secret key and ephemeral secret key are leaked to the adversary. In the case that a party's long-term secret key is leaked, only a passive adversary is assumed. The reason for this restriction is that after the long-term secret key is leaked, the adversary is obviously able to impersonate the party, which makes the adversary too powerful.

\subsection{Input to Our Verification Method}

The input assumed in our verification method is the protocol code, which consists only of the protocol specification: the formula of the variable (e.g., session key), who sent what to the communication channel, and who received what from the communication channel. Similar to TAMARIN prover, our method uses multiset rewriting rules and facts to describe the protocol. The facts corresponding to the behavior of each party are shown in Table 1.

Let the initiator and responder's names be $\$ I$ and $\$ R$, their IDs be 'Init' and 'Resp', their ephemeral secret keys be eskI and
Table 1: Corresponding Facts of the Party Behavior

\begin{tabular}{ll}
\hline Protocol specification & Fact \\
\hline $\begin{array}{l}\text { Let } k \text { be the session key for a session using the } \\
\text { ephemeral secret key esk. }\end{array}$ & Sessk $(\sim$ esk, $\mathrm{k})$ \\
Let $y$ be the variable $x$. & $\mathrm{x}=\mathrm{y}$. \\
$\begin{array}{l}\text { Send } m \text { to the communication channel. } \\
\text { Receive } m \text { from the communication channel. }\end{array}$ & Out $(\mathrm{m})$. \\
\hline
\end{tabular}

Table 2: Correspondence Between Input and Automatically Added Action Facts

\begin{tabular}{ll}
\hline Input facts & Action facts to be added \\
\hline Out $(\mathrm{m})$ & $\operatorname{Send}(\$ \mathrm{P}, \mathrm{m})$ \\
$\operatorname{In}(\mathrm{m})$ & $\operatorname{Recv}(\$ \mathrm{P}, \mathrm{m})$ \\
& $\operatorname{Accept}(\sim \operatorname{esk}, \$ \mathrm{P}, \$ \mathrm{Q}, \mathrm{skP})$ \\
$\operatorname{Sessk}(\operatorname{eskP}$, skP) & $\operatorname{Sid}\left(\sim \operatorname{eskP},<\mathrm{P}^{\prime}, \$ \mathrm{P}, \$ \mathrm{Q}, m_{\text {out }}, m_{\mathrm{in}}>\right)$ \\
& Match $\left(\sim \operatorname{eskP},<^{\prime} \mathrm{Q}^{\prime}, \$ \mathrm{Q}, \$ \mathrm{P}, m_{\mathrm{in}}, m_{\text {out }}>\right)$ \\
\hline
\end{tabular}

eskR, their long-term secret keys be $\sim 1 s k I$ and $~ 1 s k R$, their public keys be pkI and pkR, and their session keys be skI and skR, respectively. Additionally, cryptographic primitives such as symmetric key cryptography, public key cryptography, hash functions, and digital signatures are described using the same function symbols as in TAMARIN. For details of each cryptographic primitive, please refer to the official manual [33].

\subsection{Automatic Fact-Writing Algorithm}

Our verification method automatically adds the necessary facts to the security model description. This makes it possible to create a reusable security model code. The algorithm for automatically adding facts is shown below.

For a protocol code received as input, if there is an Out, In, or Sessk fact in the Pre or Post condition of each rule, the corresponding fact is added to the Action condition of that rule. The corresponding facts are shown in Table 2 . Here, $P$ means the party corresponding to the input fact (initiator I or responder R), Q means the party with which $\mathrm{P}$ communicates, $m_{\text {out }}$ means the sequence of messages that the party has sent to the communication channel so far, and $m_{\text {in }}$ means the sequence of messages that the party has received from the communication channel so far. Send and Recv facts mean that the party has sent and received a message, Accept fact means that the party has established a session key with the communication partner, Sid fact means the definition of the session ID for the session, and Match fact means the definition of the session ID of the matching session.

Then, the (not action) facts in Table 3 are automatically added to the default location regardless of the input. Here, Fr and Ephk are the facts that define the party's ephemeral secret key, and Ltk and $\mathrm{Pk}$ are the facts that define the party's long-term secret key and corresponding public key. $P_{-} i$ is a fact that is written to connect the rules of the party. 


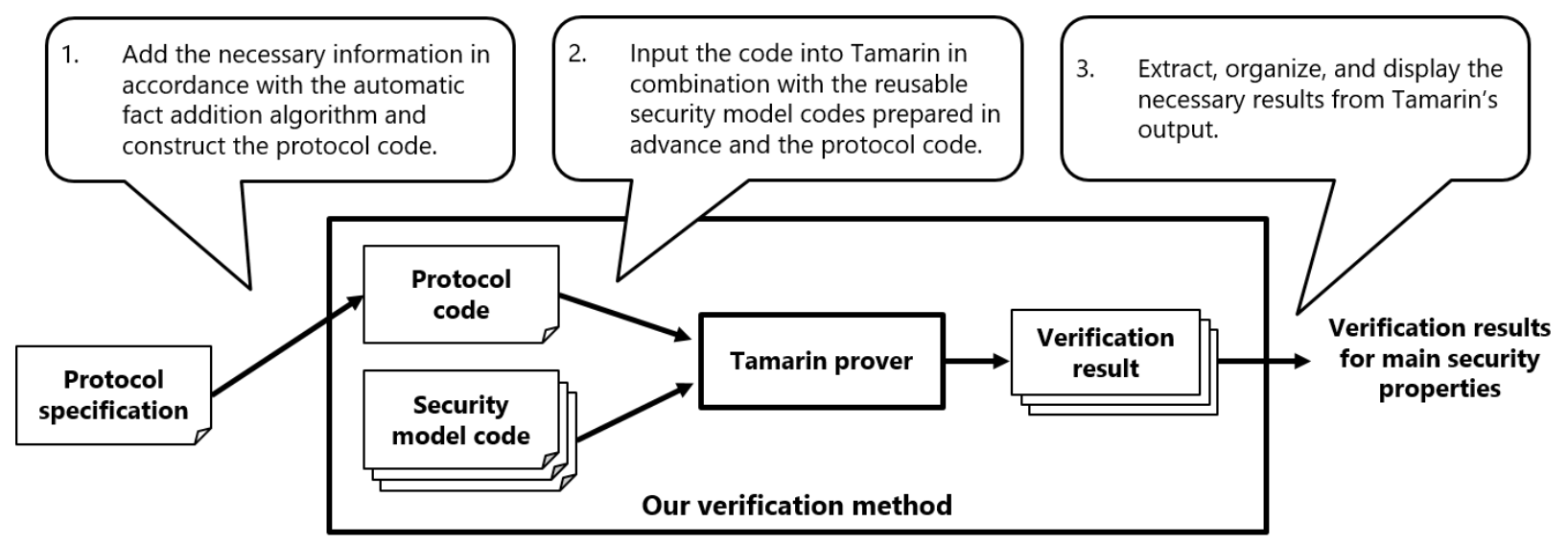

Figure 4: The conceptual diagram of our verification method.

Table 3: Facts That Are Added Regardless of the Input

\begin{tabular}{|c|c|}
\hline Location to add & Fact to add \\
\hline $\begin{array}{l}\text { Pre condition of the first } \\
\text { rule for each party }\end{array}$ & $\operatorname{Fr}(\sim \operatorname{eskP}) ! \operatorname{Ltk}(\$ P, \sim 1 s k P)$ \\
\hline $\begin{array}{l}\text { Post condition of the first } \\
\text { rule for each party }\end{array}$ & !Ephk ( eskP, eskP) \\
\hline Pre condition of each rule & $! P k(\$ Q, p k Q)$ \\
\hline Post condition of $i$ th rule & P_i $\left(\sim\right.$ eskP $\left., \$ P, \$ Q, \sim l s k P, m_{\text {out }}, m_{\text {in }}\right)$ \\
\hline Pre condition of $i+1$ th rule & $P_{-} i\left(\sim\right.$ eskP $\left., \$ P, \$ Q, \sim l s k P, m_{\text {out }}, m_{\text {in }}\right)$ \\
\hline
\end{tabular}

\subsection{Creating a Reusable Security Model Code}

In this section, we explain how we describe the security properties of Section 3.1 in a reusable TAMARIN prover input format. We use the action facts Accept, Sid, and Match to describe the security model in a unified way, and furthermore, we assume all reveal queries in advance and control the queries at the part of describing the security property to achieve reusable code.

As an example, we explain how to write the security model code for confidentiality of the session key when the responder's longterm secret key is leaked by an adversary and forward secrecy. The logical formula for the confidentiality of the session key when the responder's long-term secret key is leaked by the adversary is expressed as follows: if the adversary has the session key, then either the session key has been leaked somewhere, the initiator's long-term secret key has been leaked somewhere, the initiator or responder's ephemeral secret key has been leaked somewhere, or the responder's long-term secret key has been leaked, but the active attack has been executed. Similarly, the logical formula for the forward secrecy is expressed as follows: if the adversary has the session key, then either the session key has been leaked somewhere, the initiator or responder's ephemeral secret key has been leaked somewhere, or the initiator or responder's long-term secret key has been leaked before the session key was established. By using such a description method, we have achieved a security model that is reusable and can verify multiple security properties at once. These security model codes are shown in Appendix B.
Table 4: Verification Results That Are Theoretically Known in Cryptography and Obtained by Our Verification Method

\begin{tabular}{lcccccc}
\hline \multirow{2}{*}{ Protocol name } & \multicolumn{3}{c}{ Theoretically known } & \multicolumn{3}{c}{ Our verification method } \\
& Conf. & FS & wFS & Conf. & FS & wFS \\
\hline DH[17] & $x$ & $x$ & $\checkmark$ & $x$ & $x$ & $\checkmark$ \\
NS[30] & $x$ & $x$ & $x$ & $x$ & $x$ & $x$ \\
HMQV[26] & $\checkmark$ & $x$ & $\checkmark$ & $\checkmark$ & $x$ & $\checkmark$ \\
NAXOS[27] & $\checkmark$ & $x$ & $\checkmark$ & $\checkmark$ & $x$ & $\checkmark$ \\
CMQV[34] & $\checkmark$ & $x$ & $\checkmark$ & $\checkmark$ & $x$ & $\checkmark$ \\
\hline
\end{tabular}

\subsection{Output from Our Verification Method}

Our verification method outputs the information on which security property has been verified and what kind of information leakage would cause the session key to lose its confidentiality. For an example of the output, please refer to Appendix C.

\subsection{Validity of Our Method}

In this section, to validate of our verification method, we verify security properties of several existing protocols and show that the results coincide with results that are theoretically known in cryptography. We verify that several KE protocols satisfy the three main security properties, the confidentiality of the session key, weak forward secrecy, and forward secrecy, using the proposed method. We choose protocols to cover the pattern of security combinations that exist, but the verification of protocols that satisfy forward secrecy is a future work. The protocols used in the verification and the results of the verification by the proposed method are shown in Table 4. For details of these protocols, please refer to the cited papers. In the table, in the results that are theoretically known in cryptography, " $\checkmark$ " means that it has been proven to satisfy the security, and " $X$ " means that the security is not satisfied (attack found). In the results for our verification method, " $\checkmark$ " means no attack found, and " $X$ " means attack found by our method. We compared the results with the literature reviewed by Boyd et al. [10] and the formal verification tool by Basin and Cremers [5] and found that the results are same. 


\section{FUTURE WORK}

In the future, we plan to expand the scope of applicable cryptographic protocols. In this paper, KE protocols were the only target of verification, but they are not the only protocols that protocol designers want to verify. Therefore, we plan to expand the scope of verification to other types of cryptographic protocols (e.g., authentication protocols). To achieve this, we need to start from the formalization of security properties of other types of cryptographic protocols. In addition, we plan to improve the reliability of the verification. In this paper, we validated the verification by verifying the five main KE protocols and comparing them with the security in cryptography. We need to check the validity by using more protocols. In particular, we need to verify protocols that satisfy the forward secrecy and evaluate the proposed method. Furthermore, one of our goals is to theoretically validate the validation.

\section{REFERENCES}

[1] Nawal Zaied Almuzaini and Iftikhar Ahmad. 2019. Formal Analysis of the Signal Protocol Using the Scyther Tool. In 2019 2nd International Conference on Computer Applications Information Security (ICCAIS). 1-6. https://doi.org/10.1109/ CAIS.2019.8769532

[2] Manuel Barbosa, Gilles Barthe, Karthik Bhargavan, Bruno Blanchet, Cas Cremers, Kevin Liao, and Bryan Parno. 2021. SoK: Computer-Aided Cryptography. In SP 2020 - 42nd IEEE Symposium on Security and Privacy. Virtual Conference, United States. https://hal.inria.fr/hal-03046757

[3] David Basin, Cas Cremers, Jannik Dreier, Simon Meier, Ralf Sasse, and Benedikt Schmidt. 2021. Tamarin prover. https://tamarin-prover.github.io/. Accessed: 2021-2-5.

[4] David Basin, Jannik Dreier, Lucca Hirschi, Saša Radomirovic, Ralf Sasse, and Vincent Stettler. 2018. A formal analysis of 5G authentication. In Proceedings of the 2018 ACM SIGSAC Conference on Computer and Communications Security. 1383-1396.

[5] David A Basin and Cas JF Cremers. 2009. From Dolev-Yao to Strong Adaptive Corruption: Analyzing Security in the Presence of Compromising Adversaries. IACR Cryptol. ePrint Arch. 2009 (2009), 79.

[6] Karthikeyan Bhargavan, Bruno Blanchet, and Nadim Kobeissi. 2017. Verified Models and Reference Implementations for the TLS 1.3 Standard Candidate. In IEEE Symposium on Security and Privacy (S\&P'17). IEEE, San Jose, CA, 483-503. Distinguished paper award.

[7] Bruno Blanchet. 2013. Automatic verification of security protocols in the symbolic model: The verifier proverif. In Foundations of security analysis and design VII. Springer, 54-87.

[8] Bruno Blanchet. 2016. Modeling and verifying security protocols with the applied pi calculus and ProVerif. Foundations and Trends® in Privacy and Security 1, 1-2 (2016), 1-135.

[9] Bruno Blanchet. 2021. ProVerif: Cryptographic protocol verifier in the formal model. https://prosecco.gforge.inria.fr/personal/bblanche/proverif/. Accessed: 2021-2-5.

[10] Colin Boyd, Anish Mathuria, and Douglas Stebila. 2019. Protocols for authentication and key establishment Second Edition. Springer.

[11] Iliano Cervesato, Nancy A Durgin, Patrick D Lincoln, John C Mitchell, and Andre Scedrov. 1999. A meta-notation for protocol analysis. In Proceedings of the 12th IEEE Computer Security Foundations Workshop. IEEE, 55-69.

[12] Cas Cremers. 2021. The Scyther Tool. https://people.cispa.io/cas.cremers/ scyther/. Accessed: 2021-2-5.

[13] Cas Cremers and Martin Dehnel-Wild. 2019. Component-Based Formal Analysis of 5G-AKA: Channel Assumptions and Session Confusion. In Network and Distributed Systems Security (NDSS) Symposium 2019. https://publications.cispa. saarland/2758/

[14] Cas Cremers, Marko Horvat, Jonathan Hoyland, Sam Scott, and Thyla van der Merwe. 2017. A comprehensive symbolic analysis of TLS 1.3. In Proceedings of the 2017 ACM SIGSAC Conference on Computer and Communications Security. $1773-1788$.

[15] Cas Cremers, Marko Horvat, Sam Scott, and Thyla van der Merwe. 2016. Automated analysis and verification of TLS 1.3: 0-RTT, resumption and delayed authentication. In 2016 IEEE Symposium on Security and Privacy (SP). IEEE, 470485.

[16] Cas JF Cremers. 2008. The Scyther Tool: Verification, falsification, and analysis of security protocols. In International conference on computer aided verification. Springer, 414-418.
[17] Whitfield Diffie and Martin Hellman. 1976. New directions in cryptography. IEEE transactions on Information Theory 22, 6 (1976), 644-654.

[18] Danny Dolev and Andrew Yao. 1983. On the security of public key protocols IEEE Transactions on information theory 29, 2 (1983), 198-208.

[19] Zoom Video Communications Inc. 2021. Video Conferencing, Web Conferencing, Webinars, Screen Sharing - Zoom. https://zoom.us/. Accessed: 2021-2-5.

[20] Zoom Video Communications Inc. 2021. Zoom End-to-End Encryption Whitepaper. https://github.com/zoom/zoom-e2e-whitepaper. Accessed: 20212-5.

[21] Michel Keller. 2014. Converting alice\&bob protocol specifications to tamarin. (2014). Bachelor's thesis, ETH Zurich, https://ethz.ch/ content/dam/ethz/special-interest/infk/inst-infsec/information-securitygroup-dam/research/software/thesis_keller_alicebob.pdf.

[22] Jun Young Kim, Ralph Holz, Wen Hu, and Sanjay Jha. 2017. Automated analysis of secure internet of things protocols. In Proceedings of the 33rd Annual Computer Security Applications Conference. 238-249.

[23] Nadim Kobeissi. 2021. Verifpal. https://verifpal.com/. Accessed: 2021-2-5.

[24] Nadim Kobeissi, Karthikeyan Bhargavan, and Bruno Blanchet. 2017. Automated Verification for Secure Messaging Protocols and Their Implementations: A Symbolic and Computational Approach. In 2017 IEEE European Symposium on Security and Privacy (EuroS P). 435-450. https://doi.org/10.1109/EuroSP.2017.38

[25] Nadim Kobeissi, Georgio Nicolas, and Mukesh Tiwari. 2020. Verifpal: Cryptographic Protocol Analysis for the Real World. In Proceedings of the 2020 ACM SIGSAC Conference on Cloud Computing Security Workshop. 159-159.

[26] Hugo Krawczyk. 2005. HMQV: A high-performance secure Diffie-Hellman protocol. In Annual International Cryptology Conference. Springer, 546-566.

[27] Brian LaMacchia, Kristin Lauter, and Anton Mityagin. 2007. Stronger security of authenticated key exchange. In International conference on provable security. Springer, 1-16.

[28] Bill Marczak and John Scott-Railton. 2020. Move Fast and Roll Your Own Crypto: A Quick Look at the Confidentiality of Zoom Meetings. Citizen Lab Research Report No. 126, University of Toronto.

[29] Simon Meier, Benedikt Schmidt, Cas Cremers, and David Basin. 2013. The TAMARIN prover for the symbolic analysis of security protocols. In International Conference on Computer Aided Verification. Springer, 696-701.

[30] Roger M Needham and Michael D Schroeder. 1978. Using encryption for authentication in large networks of computers. Commun. ACM 21, 12 (1978), 993-999.

[31] Benedikt Schmidt, Simon Meier, Cas Cremers, and David Basin. 2012. Automated Analysis of Diffie-Hellman Protocols and Advanced Security Properties. In 2012 IEEE 25th Computer Security Foundations Symposium. 78-94. https://doi.org/10. 1109/CSF.2012.25

[32] Gang Shen, Xiaohong Li, Ruitao Feng, Guangquan Xu, Jing Hu, and Zhiyong Feng. 2014. An extended uml method for the verification of security protocols. In 2014 19th International Conference on Engineering of Complex Computer Systems. IEEE, 19-28.

[33] The Tamarin Team. 2021. Tamarin-Prover Manual Security Protocol Analysis in the Symbolic Model. https://tamarin-prover.github.io/manual/tex/tamarinmanual.pdf. Accessed: 2021-2-5.

[34] Berkant Ustaoglu. 2008. Obtaining a secure and efficient key agreement protocol from (H) MQV and NAXOS. Designs, Codes and Cryptography 46, 3 (2008), 329342.

[35] Jorden Whitefield, Liqun Chen, Frank Kargl, Andrew Paverd, Steve Schneider, Helen Treharne, and Stephan Wesemeyer. 2017. Formal analysis of V2X revocation protocols. In International Workshop on Security and Trust Management. Springer, 147-163.

[36] Jorden Whitefield, Liqun Chen, Ralf Sasse, Steve Schneider, Helen Treharne, and Stephan Wesemeyer. 2019. A symbolic analysis of ecc-based direct anonymous attestation. In 2019 IEEE European Symposium on Security and Privacy (EuroS\&P). IEEE, 127-141.

[37] Jingjing Zhang, Qiang Wang, Lin Yang, and Tao Feng. 2019. Formal Verification of 5G-EAP-TLS Authentication Protocol. In 2019 IEEE Fourth International Conference on Data Science in Cyberspace (DSC). 503-509. https://doi.org/10.1109/ DSC. 2019.00082

[38] Jingjing Zhang, Lin Yang, Weipeng Cao, and Qiang Wang. 2020. Formal Analysis of 5G EAP-TLS Authentication Protocol Using Proverif. IEEE Access 8 (2020), 23674-23688. https://doi.org/10.1109/ACCESS.2020.2969474

[39] Jingjing Zhang, Lin Yang, Xianming Gao, Gaigai Tang, Jiyong Zhang, and Qiang Wang. 2020. Formal analysis of QUIC handshake protocol using ProVerif. In 2020 7th IEEE International Conference on Cyber Security and Cloud Computing (CSCloud)/2020 6th IEEE International Conference on Edge Computing and Scalable Cloud (EdgeCom). 132-138. https://doi.org/10.1109/CSCloudEdgeCom49738.2020.00030

[40] Jingjing Zhang, Lin Yang, Xianming Gao, Gaigai Tang, Jiyong Zhang, and Qiang Wang. 2021. Formal Analysis of QUIC Handshake Protocol Using Symbolic Model Checking. IEEE Access 9 (2021), 14836-14848. https://doi.org/10.1109/ ACCESS.2021.3052578 


\section{A EXAMPLE OF INPUT OF TAMARIN}

As an example of input of TAMARIN, the protocol code of the DH key-exchange protocol (see Figure 3) and the corresponding security model code for confidentiality of the session key when a party's ephemeral secret key (random number) is leaked by an adversary are shown below.

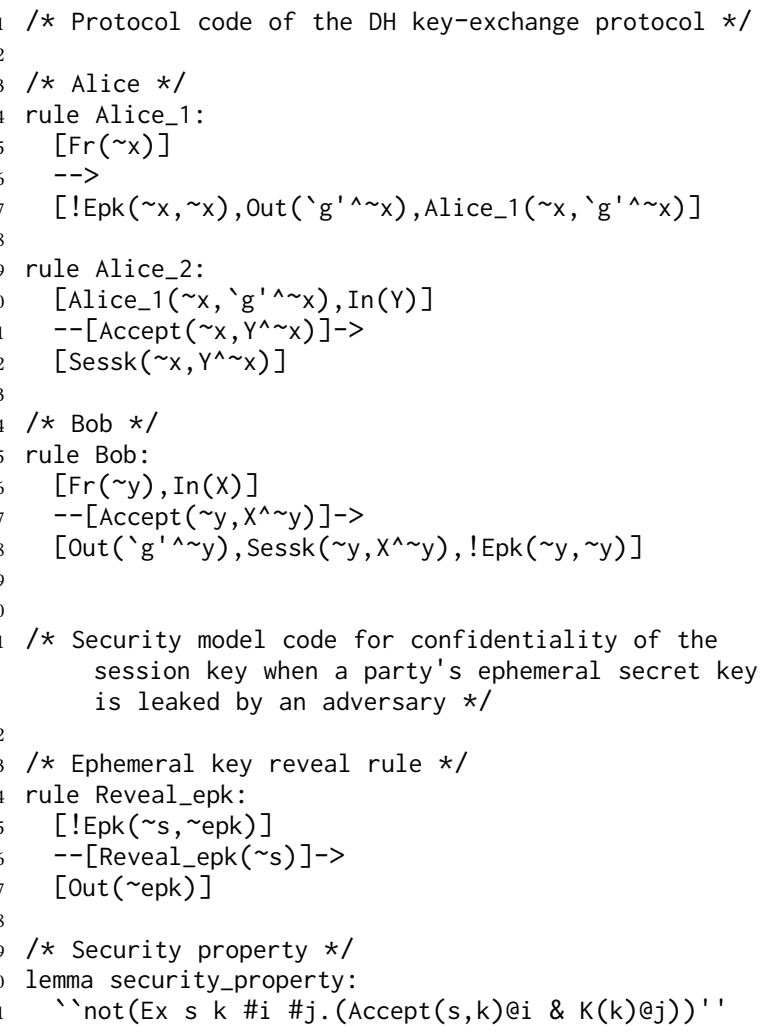

In the protocol code, lines 3 to 12 represent Alice's actions: generate a random number $x(\operatorname{Fr}(\sim x))$, send $g^{x}$ to the communication channel (Out (' $g$ '^ $\sim \mathrm{x}$ )), receive $Y\left(=g^{y}\right.$ ) from the channel $(\operatorname{In}(Y))$, and set the session key to $Y^{x}\left(=g^{x y}\right)\left(\operatorname{Sessk}\left(\sim \mathrm{X}, \mathrm{Y}^{\wedge} \sim \mathrm{X}\right)\right)$. Similarly, lines 14 to 18 represent Bob's action: generate a random number $y(\operatorname{Fr}(\sim y))$, send $g^{y}$ to the communication channel (Out (' $g$ '` $\sim y)$ ), receive $X\left(=g^{x}\right)$ from the channel $(\operatorname{In}(X))$, and set the session key to $X^{y}\left(=g^{x y}\right)\left(\operatorname{Sessk}\left(\sim y, X^{\wedge} \sim y\right)\right)$.

In the security model code, lines 23 to 27 represent the leakage of the party's ephemeral secret key, and lines 29 to 31 represent the confidentiality of the session key, where $\operatorname{Accept}(s, k) @_{i} \&$ $K(k) @ j$ means that the adversary knows the session key $k$ of session $s$ at time $i$ at time $j$.

In TAMARIN, the variable name needs to be prefixes with symbols $\sim$, \$, or \# depending on the type of variable. Newly declared variables such as random number information should be prefixed with $\sim$, public information such as the name of the party that should be prefixed with $\$$, and variables representing a point in time should be prefixed with \#. Other variables such as messages are not marked with any symbol. Each security property is described by the name of lemma (as in lemma security_property: in line 30), and the result is output for each lemma.

\section{B EXAMPLE OF REUSABLE SECURITY MODEL CODE}

As an example of reusable security model code, we show below the security model code for confidentiality of the session key when the responder's long-term secret key is leaked by an adversary and forward secrecy.

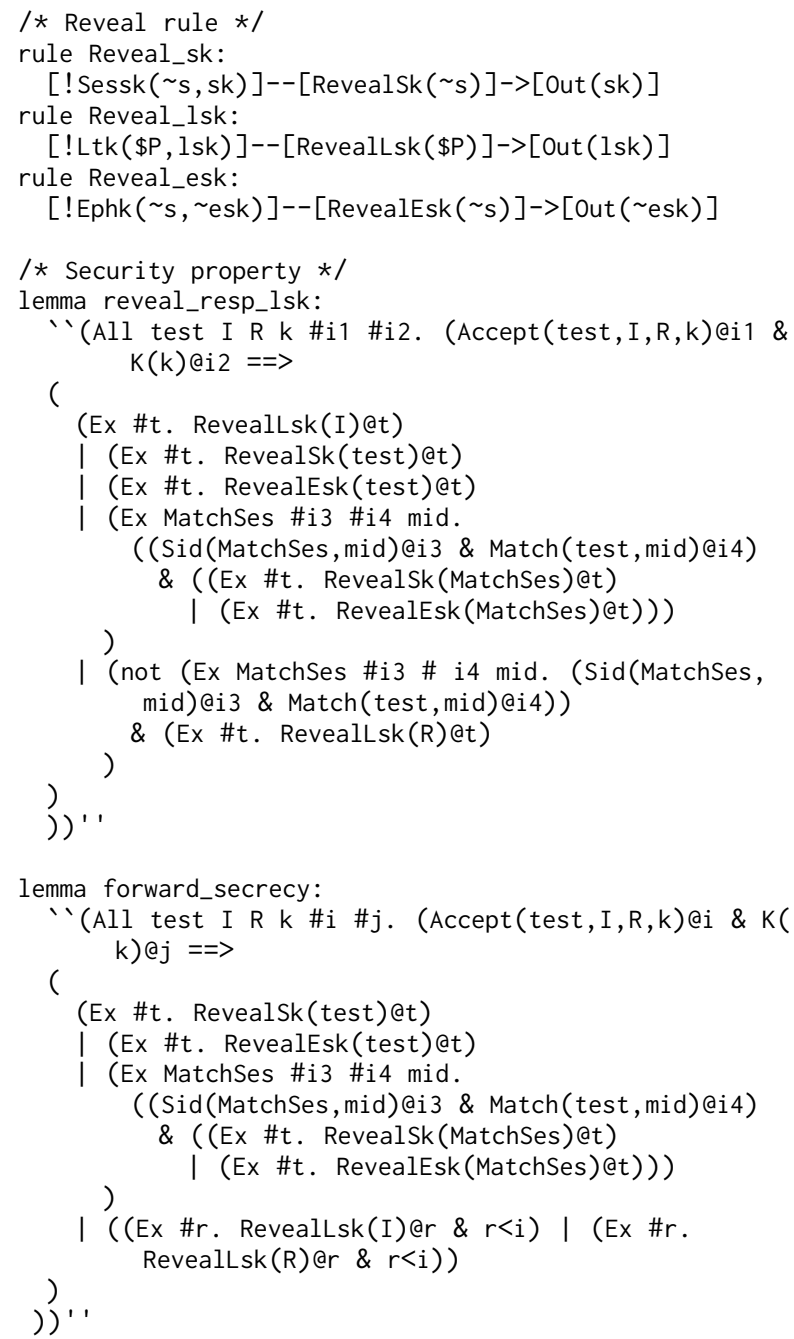

We use the action facts Accept, Sid, and Match to describe the security model in a unified way, and furthermore, we assume all reveal queries in advance and control the queries at the part of describing the security property to achieve reusable code.

In the above security model code, lines 2 to 7 represent the leakage of the party's information, lemma reveal_resp_lsk from lines 10 to 25 represents the confidentiality of the session key when the responder's long-term secret key is leaked by the adversary, and lemma forward_secrecy from lines 27 to 38 represents the forward secrecy. 


\section{EXAMPLE OF VERIFICATION BY OUR METHOD}

In this section, as an example of verification using our proposed method, we show the case of verifying the DH KE protocol (see Figure 3). The input code is as follows:

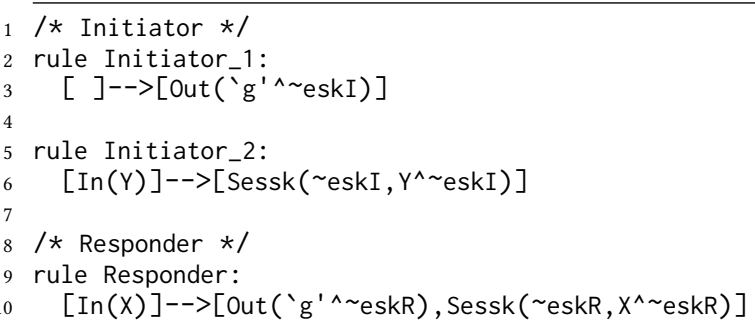

When the above code is input, facts are added in accordance with the automatic fact addition algorithm described in Section 3.3, and the following protocol code is generated:

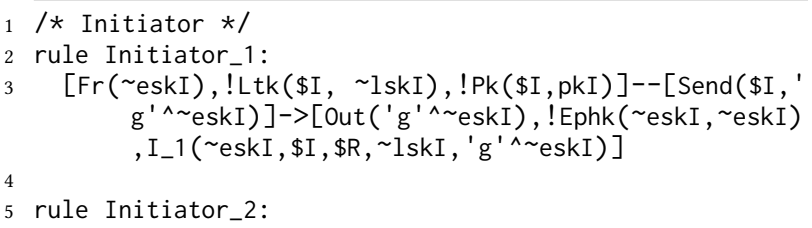

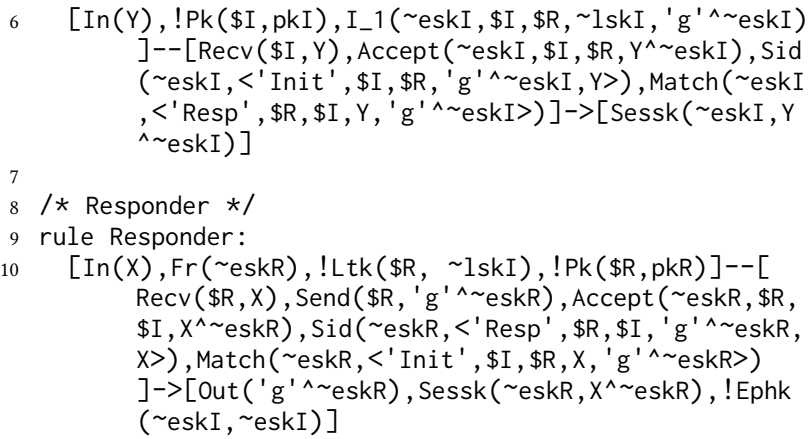

Then, verification against the defined security model is performed automatically, and the following output is displayed:

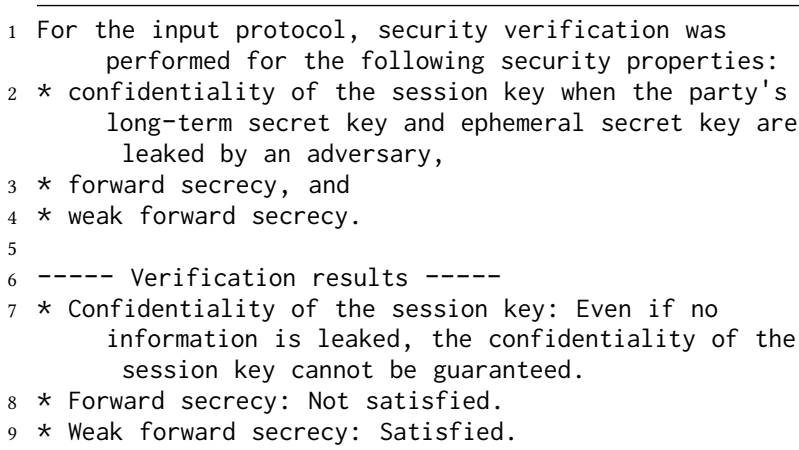

